\section{Luftverschmutzung reduziert positive Gesundheitseffekte des Spazierengehens}

Sinharay R et al. Respiratory and cardiovascular responses to walking down a traffic-polluted road compared with walking in a traffic-free area in participants aged 60 years and older with chronic lung or heart disease and age-matched healthy controls: a randomised crossover study. Lancet 2018; 391: 339 - 349

doi:10.1016/S0140-6736(17)32643-0

Feinstäube und Stickstoffdioxid können eine COPD und KHK nachteilig beeinflussen. Auf der anderen Seite steigern Spaziergänge an der frischen Luft nicht nur das seelische Wohlbefinden, sondern wirken sich auch auf körperliche Symptome positiv aus. Die Crossover-Studie verglich die Effekte eines langen Spaziergangs an einer abgasbelasteten Straße und im Park bei Patienten und Gesunden.

An der Studie nahmen 40 gesunde Freiwillige, 40 Patienten mit einer chronisch obstruktiven Lungenerkrankung (COPD) und 39 Patienten mit einer koronaren Herzerkrankung (KHK) teil. Einschlusskriterien waren u.a. ein GOLD-Stadium 2 , eine $\mathrm{FEV}_{1} \leq 80 \%$ bzw. koronarangiografisch belegte Koronarstenosen, eine seit 6 Monaten stabile Krankheitsphase, eine $\geq 12$-monatige Nikotinabstinenz und ein Alter $\geq 60$ Jahre. Nach Basisuntersuchungen in einem Londoner Krankenhaus spazierten die Teilnehmer entweder 2 Stunden entlang der durch Taxis und Busse stark mit Dieselabgasen belasteten Oxford Street oder im verkehrsfreien Hyde Park. Vorgesehen war eine Strecke von etwa $5 \mathrm{~km}$ von 11-13 Uhr. Während des Laufens wurde die Belastung durch Rußpartikel, Feinstaub $\left(\mathrm{PM}_{2,5}\right.$ und $\left.\mathrm{PM}_{10}\right)$, Ultrafeinstaub, Stickstoffdioxid $\left(\mathrm{NO}_{2}\right)$ und Lärm gemessen. Nach dem Gang erfolgten weitere 24 Stunden Verlaufsuntersuchungen der Lungenfunktion, arteriellen Gefäßflexibilität und klinischen Symptome. Nach 3-8 Wochen gingen die Teilnehmer die alternative Strecke, erneut mit Vor- und Nachuntersuchungen.

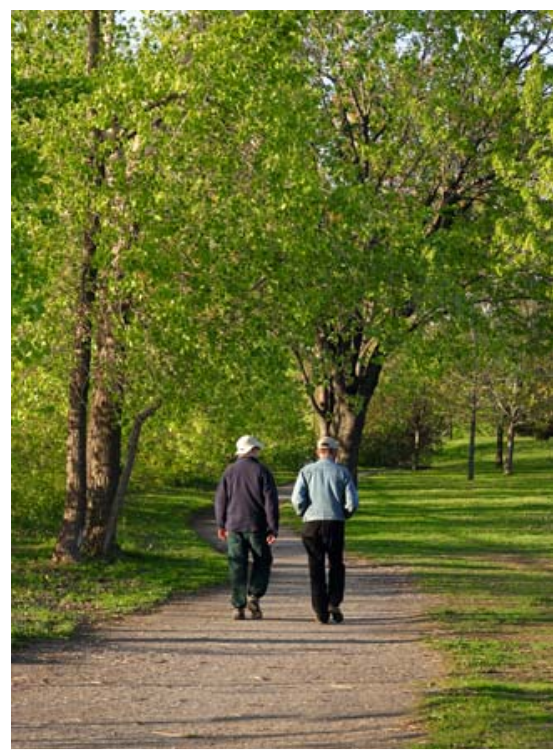

- Spaziergänge in frischer Luft wirkten sich positiv auf die pulmonale und vaskuläre Gesundheit von Patienten mit COPD und KHK aus. In der abgasbelasteten Innenstadt nahmen die günstigen Effekte ab. Symbolbild; Bildnachweis: istockphoto.

Bei allen Personen verbesserte die Bewegung im Park unabhängig vom Gesundheitsstatus die Lungenfunktion ( $\mathrm{FEV}_{1}$ und FVC) und verminderte die Pulswellengeschwindigkeit und den Augmentationsindex als Maß der Gefäßsteifigkeit. Die Effekte bestanden auch am Ende des Interventionstages nach 26 Stunden. Die positiven Gesundheitseffekte des Spazierengehens waren an der verkehrsbelasteten Straße deutlich abgeschwächt. Verglichen mit dem Park hatten Patienten mit COPD nach der Oxford Street stärkeren Husten, Auswurf und Atemnot. Patienten mit KHK gaben stärkeren Husten an. Die Kontrollen bemerkten keine subjektiven Unterschiede. Die Belastung durch spezifische Luftbestandteile beeinflusste die Lungenfunktion und Gefäßsteifigkeit. Bei Patienten mit einer COPD korrelierten eine verminderte FEV1 und FVC 3 Stunden nach der Oxford Street signifikant mit der Konzentration von $\mathrm{PM}_{2,5}$, Ultrafeinstaub und $\mathrm{NO}_{2}$. Eine reduzierte FVC war mit zunehmenden $\mathrm{PM}_{10}$ und der Lärmexposition assoziiert. In der 4-Stunden-Untersuchung korrelierte ein gestiegener Atemwegwiderstand mit $\mathrm{PM}_{2,5}$, Rußpartikeln, Ultrafein- staub, $\mathrm{NO}_{2}$ und $\mathrm{PM}_{10}$. Bei Patienten mit KHK und Gesunden bestanden keine signifikanten Assoziationen von Luftverschmutzungsgrad und Lungenfunktion. Analog dazu ergab sich eine Beziehung zwischen einer verminderten Gefäßelastizität und der Luftbelastung durch Rußpartikel, Ultrafeinstaub und $\mathrm{NO}_{2}$ nur bei Patienten mit COPD. Nach Subgruppenanalysen führten die Autoren die ausbleibende Assoziation bei KHK auf die Medikation der Patienten zurück, die offensichtlich modulierende Effekte hatte. Rauchen beeinflusste die Ergebnisse nicht.

FAZIT

Spaziergänge in frischer Luft wirkten sich positiv auf die pulmonale und vaskuläre Gesundheit aus. In der belasteten Innenstadt nahmen günstige Effekte auf die Lungengesundheit und Gefäßflexibilität ab. Patienten mit KHK waren vaskulär möglicherweise durch Medikamente geschützt, so die Autoren. Die Wissenschaftler empfehlen für Patienten mit COPD oder KHK 3 Dinge: Spaziergänge in der unbelasteten Natur, Vermeidung von längeren Innenstadtaufenthalten und eine kardioprotektive Medikation bei KHK.

Dr. med. Susanne Krome, Melle 